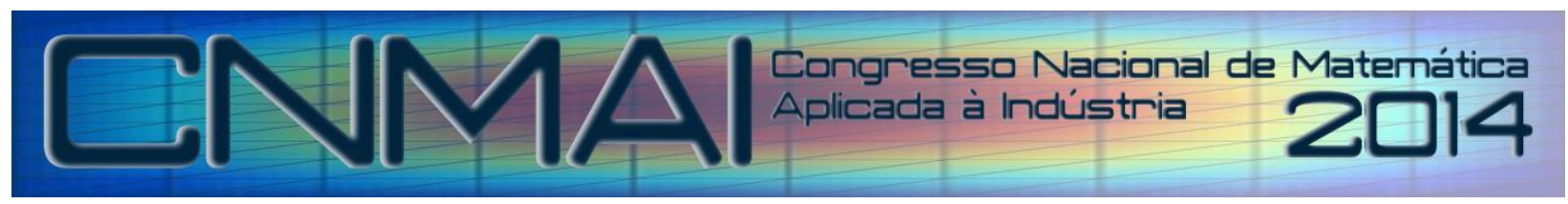

18 a 21 de novembro de 2014, Caldas Novas - Goiás

\title{
USO DA EQUAÇÃO DE HALL-PETCH PARA PREVER AS PROPRIEDADES MECÂNICAS DE PONTOS DA ZONA TERMICAMENTE AFETADA PELA SOLDAGEM DO AÇO API 5L X80, ATRAVÉS DE AMOSTRAS PRODUZIDAS NO SIMULADOR TERMOMECÂNICO
}

\author{
Roberto de Araújo Alécio, ralecio@gmail.com; \\ Jorge Luís Lauriano Gama, Jorge.lauriano@gmail.com²; \\ Tiago de Sousa Antonino, tiago_antonino@yahoo.com ${ }^{3}$; \\ Pablo Batista Guimarães, pabloguimaraes@ifpe.recife.edu.br³ \\ José Maria Andrade Barbosa, jmab13@ufpe.br ${ }^{1}$; \\ Yogendra Prasad Yadava, yadava@ufpe.br ${ }^{1}$; \\ Ricardo Artur Sanguinetti Ferreira, ras@ufpe.br¹. \\ ${ }^{1}$ Departamento de Engenharia Mecânica/Centro de Tecnologia e Geociencias/UFPE \\ Av. Prof. Moraes Rego, 1235 - Cidade Universitária, CEP: 50670-901, Recife/PE. \\ ${ }^{2}$ Coordenação do Curso de Mecânica/Gerência Acadêmica de Tecnologia e Processos/IFAL \\ Av. Barão de Atalaia, s/n - Centro, CEP: 57050-520, Maceió/AL. \\ ${ }^{3}$ Coordenação de Mecânica/Departamento Academico de Controle e Processos Industriais/ /IFPE \\ Av. Prof Luiz Freire, 500, - Cidade Universitária, CEP: 50740-540 Recife/PE.
}

Resumo: A Zona termicamente Afetada (ZTA) de um aço submetido a soldagem (pequena região que situa-se na viziança da zona de fusão $(Z F)$ onde há um grande aquecimento sem fundir) é sub-dividida em função do ciclo térmico ( pico de temperatura) que lhe é atingido. O tamanho de grão é um parâmetro microestrutural básico para se obter as propriedades mecânicas que, associando o ciclo térmico a diagrama de transformação de fase, observa-se na ZTA uma microestrutura de grãos grosseiros logo após a zona de ligação, a seguir uma região de grão finos, depois uma região intercrítica e por fim uma região Subcrítica que recebe aquecimento menor que $973 K$. A equação empírica de Hall-Petch relaciona a tensão de escoamento de um metal com o diametro médio de grão, com base em medições experimentais, conhecendo as constantes relacionadas ao material de interesse. É comum a perda de propriedades na ZTA em virtude de transformação microestrutural indesejável (fragilização, precipitação, etc.) devido os ciclos termicos. Uma metodologia teórico-experimental que possibilita a produção de microestruturas, está sendo desenvolvida neste trabalho, a partir de resfriamento induzido num simulador termomecânico, reproduzindo as condições termodinâmicas de sub-regiões das ZTAs assegurando a homogeneidade microestrutural em corpos de provas com dimensões normalizadas. São analisados os dados experimentais segundo a equação empírica de HallPetch, de modo que as variáveis de tensão de atrito e coeficiente particular do material são obtidas por regressão linear, com a curva definida a partir dos valores de resistência e tamanho de grãos do corpo de prova (CP) produzido no simulador termomecânico. Baseados na caracterização microestrutural foi realizada uma junta soldada $e$ identificados pontos da ZTA equivalentes microestruturalmente aos corpos de provas produzidos no simulador termomecânico. Considerando-se a relação de Hall-Petch foram comparadas as tensões simuladas pelo modelo matemático comparando-as às tensões dos pontos da ZTA da junta soldada. A metodologia foi conclusiva satisfatória, pois, mostrou que, das observações micrográficas e os valores de tensão obtidas confirmam o que define a literatura sobre o aço em estudo.

Palavras-chave: Hall-Petch, Propriedades Mecânicas, Simulador Termomecânico, ZTA.

\section{INTRODUÇÃO}

A necessidade permanente do aumento quantitativo no consumo de petróleo e seus derivados têm motivado, entre outros, o estudo e desenvolvimento de materiais para utilização em transporte dutoviário que possam apresentar 
melhores propriedades mecânicas. Os Aços designados como de Alta Resistência e Baixa Liga-ARBL vem atendendo ao longo do tempo a essa demanda. O estudo de melhorias nas propriedades mecânicas dessa classe de materiais objetiva a redução do peso através da diminuição da espessura de parede, com projetos de tubos mais finos, mais resistentes e com melhor tenacidade, acompanhado consequentemente, do estudo para uma melhor soldabilidade, possibilitando uma redução no custo por soldagem e transporte, atendendo assim, as exigências de mercado.

Uma metodologia teórico-experimental que possibilita a produção de microestruturas de sub-regiões da ZTA a partir de resfriamento induzido num simulador termomecânico, assegurando a homogeneidade microestrutural num corpo de prova com dimensões normalizadas, está sendo desenvolvida neste trabalho, permitindo a determinação das propriedades mecânicas em diferentes regiões da ZTA.

As condições termodinâmicas de alguns pontos da ZTA, de acordo com as temperaturas especificadas, foram simuladas para reproduzir as condições reais de processos de soldagem GMAW (Gas Metal Arc Welding) de modo a tornar evidente as microestruturas e as propriedades mecânicas, onde essa análise realizada nestes corpos de prova visa obter valores de tensão e quantificar tamanho médio de grãos de cada uma das regiões.

Os resultados mostraram que o tamanho de grão é reduzido na medida em que nos afastamos da zona de ligação e, quanto maior for o tamanho de grão menor será a resistência mecânica. Para comparação com os valores do CP (corpo de prova) produzido no simulador termomecânico, foi produzida pelos processos GMAW uma junta soldada para identificar pontos da ZTA equivalentes microestruturalmente aos CPs produzidos no simulador termomecânico.

São analisados também os dados experimentais para validação de um modelo matemático segundo a relação empírica de Hall-Petch, de modo que as variáveis de tensão de atrito e coeficiente particular do material foram obtidas por regressão linear com a curva definida a partir dos valores de resistência e tamanho de grãos do CP produzido no simulador termomecânico.

Os resultados observados para o material em estudo, baseado na quantificação do tamanho de grão da ZTA da junta soldada e do material produzido no simulador termomecânico, demonstram a viabilidade de utilização do simulador para a discretização de pontos da ZTA de uma junta soldada, permitindo que suas propriedades mecânicas possam ser determinadas por ensaios tradicionais.

Considerando-se a relação de Hall-Petch foram comparadas as tensões simuladas pelo modelo matemático comparando-as às tensões dos pontos da ZTA da junta soldada. Controlando-se as condições termodinâmicas no simulador é possível obter-se materiais com microestrutura e tamanho de grão comparáveis a pontos discretos da ZTA que poderá servir de base para modelagem das propriedades mecânicas da junta.

A metodologia aplicada foi conclusiva satisfatória, pois, mostrou que, das observações micrográficas e dos valores calculados de tamanhos de grãos, confirmam o que define a literatura sobre o aço API 5L X80 e suas propriedades mecânicas.

\section{MATERIAIS E MÉTODOS}

No presente estudo foi utilizado o aço API 5L X80 com composição química analisada por espectrometria de emissão ótica, indicado na tab. 1.

Tabela 1. Composição química, \% em peso.

\begin{tabular}{|c|c|c|c|c|c|c|c|c|c|c|c|}
\hline $\mathrm{C}$ & $\mathrm{Si}$ & $\mathrm{Mn}$ & $\mathrm{P}$ & $\mathrm{S}$ & $\mathrm{Cr}$ & $\mathrm{Mo}$ & $\mathrm{Ni}$ & $\mathrm{Cu}$ & $\mathrm{Nb}$ & $\mathrm{Ti}$ & $\mathrm{V}$ \\
\hline 0,08 & 0,28 & 1,80 & 0,021 & 0,003 & 0,15 & 0,20 & 0,02 & 0,01 & 0,062 & 0,016 & 0,021 \\
\hline
\end{tabular}

Para a junta soldada, foi extraída duas chapas com dimensões de $150 \times 80 \times 7,5 \mathrm{~mm}$ preparada com bisel de $25^{\circ}$. A soldagem foi realizada empregando-se o processo MAG (Metal Active Gás), usando-se CO2 como gás de proteção e metal de adição na forma de arame de especificação AWS 5.18 ER70S-6 com 1,20 mm diâmetro. A amostra para microscopia, apresentadas na fig. 1, foi extraída da junta soldada, preparada por metalografia convencional conforme NBR 13284 (1995), com uma sequência de corte, lixamento e polimento, empregando lixas de granulometrias 280, 400, 600, 1000 e 1200 mesh e polimento em tapete com pasta diamantada de $1 \mu \mathrm{m}$ e após atacadas com Nital a $5 \%$ por 20 segundos. NBR 8108 (1983).

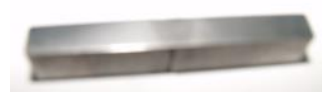

Figura 1. Amostra retirada da junta soldada para microscopia.

- As amostras foram caracterizadas por microscopia ótica em microscópio ótico Olympus Modelo BX 51M, utilizando o software Analysis. A estimativa do tamanho dos grãos foi realizada pelo método do intercepto, conforme a 
norma ASTM E-1122 (1989). Pela medição em microscópio ótico, foi observado uma ZTA de 4,6 mm e dividida em cinco regiões para observar e obter o valor do tamanho médio do grão como mostrado na fig. 2.

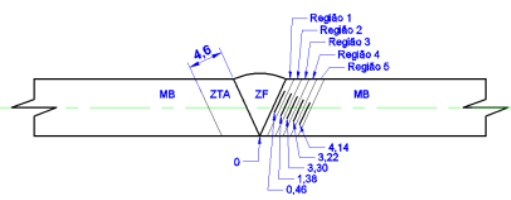

Figura 2. Sub-regiões onde foram medidos os tamanhos médios de grãos .

A simulação dos ciclos térmicos de soldagem foi desenvolvida um equipamento capaz de reproduzir resfriamentos rápidos. O equipamento é constituído de dois blocos de aço, como mostrado na fig. 3, que atuam como substrato de massa infinita; possibilitando a retirada de calor a partir de diferentes temperaturas. Silva et al (2010).

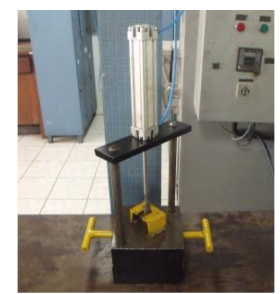

Figura 3. Simulador termomecânico.

Para os ensaios foram produzidas três amostras de acordo com as normas para método de ensaio à temperatura ambiente em materiais metálicos, Ensaio de Tração (ABNT NBR ISO 6892-1:2013), como apresentadas na fig. 4, esses corpos de prova produzidos em simulador termomecânico foram aquecidos num forno tipo mufla por 10 minutos depois de estabilizado, a diferentes temperaturas no domínio austenítico $\left(1100,1000\right.$ e $\left.900^{\circ} \mathrm{C}\right)$ e depois resfriados entre as placas do substrato do simulador. Durante o resfriamento entre os blocos do simulador, os corpos de prova são submetidos a esforços compressivos e cisalhantes, tal como aconteceria numa junta soldada. Para os ensaios de tração utilizou-se uma máquina universal de ensaios, eletromecânica e microprocessada, marca EMIC modelo DL10000, com velocidade de deslocamento de $1 \mathrm{~mm} / \mathrm{min}$ e célula de carga com capacidade máxima de até $10000 \mathrm{kgf}$.

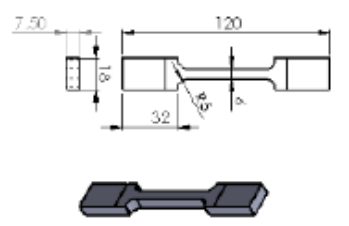

Figura 4. Corpo de prova produzido em simulador termomecânico para teste de tração. NBR 6152 (2002).

Para comparação com resultados obtidos nas amostras produzidas pelo simulador, foi realizado uma junta soldada realizada pelo processo GMAW, onde o tipo de transferência vai estar essencialmente influenciado pela intensidade de corrente, Castro et al (2012), assim as microestruturas da ZTA do cordão de solda foram comparadas com as microestruturas do material produzidos no simulador.

As condições termodinâmicas de alguns pontos de zonas termicamente afetada foram simuladas para reproduzir as condições reais de processos de soldagem, de modo a tornar evidente as microestruturas e as propriedades mecânicas em pontos discretos destas ZTAs, mas reproduzidas em corpos de prova da maior extensão. A metodologia metalográfica para determinação do tamanho de grão seguiu a mesma metodologia realizada para a junta soldada anteriormente descrita.

\section{RESULTADOS E DISCUSSÃO}

O endurecimento do aço por refino de grão é um mecanismo que melhora as propriedades do aço, Ginzburg (2003). $\mathrm{O}$ endurecimento por refino de tamanho de grão é quantificado pela equação de Hall-Petch, sendo proporcional ao inverso da raiz quadrada do diâmetro médio dos grãos através da eq. 1.

$$
\Delta \sigma_{t g}=\frac{k_{t g}}{\sqrt{d}}
$$


Onde " $\Delta \sigma_{\mathrm{tg}}$ " representa a diferença entre a tensão do metal na condição estudada e o na condição como recebido, " $\mathrm{k}_{\mathrm{tg}}$ " é uma constante que depende de característica intrínseca do metal e "d" é o tamanho de grão. A fig. 5 mostra essas várias regiões da ZTA em uma solda feita com um passe.

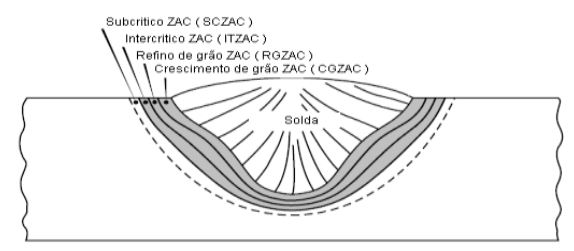

Figura 5. Várias regiões da ZTA em um cordão de solda. Ginzburg (2003).

Os valores de tamanhos médios de grãos, conforme observações realizadas em microscópio ótico, obtidos em cada região da ZTA do corpo de prova da junta soldada estão quantificados na fig. 6 para as cinco regiões destacadas. Na região R1 o tamanho foi de $0,120 \mathrm{~mm}$, na R2 de 0,092 mm, na R3 de 0,088 mm, na R4 de 0,064 mm, na R5 de 0,024 $\mathrm{mm}$ e no $\mathrm{MB}$ o valor foi de $0,020 \mathrm{~mm}$.

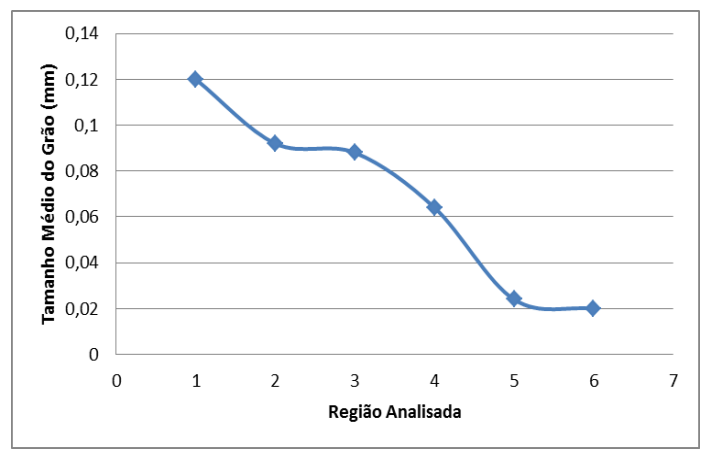

Figura 6 - Tamanho médio de grão em cada sub-região da ZTA da junta soldada.

A figura 7 apresenta as microscopias observadas nas amostras produzidas no simulador termomecânico nas diferentes temperaturas de aquecimento. Para todas as temperaturas simuladas pode ser visualizado, com 500x de aumento, o refino de grão a medida que se vai direcionando para o metal de base.

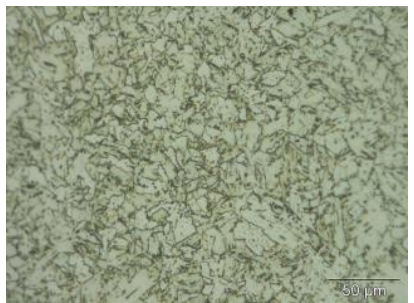

(a)

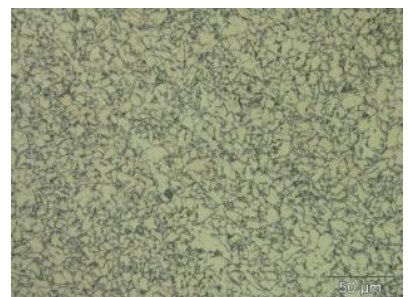

(b)

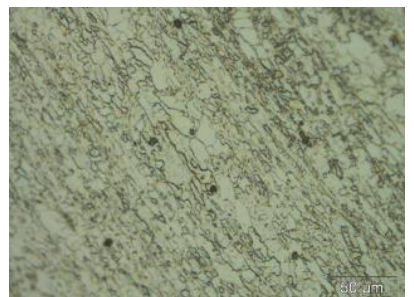

(c)

Figura 7. Microscopia do corpo de prova simulado. (a) aquecido à $1100{ }^{\circ} \mathrm{C}$; (b) aquecido à $1000{ }^{\circ} \mathrm{C} e$; (c) aquecido à $900{ }^{\circ} \mathrm{C}$.

Nas estruturas produzidas no simulador termomecânico obteve um tamanho médio de grão de $0,048 \mathrm{~mm}$ para a amostra aquecida a $1100^{\circ} \mathrm{C}, 0,028 \mathrm{~mm}$ para a amostra aquecida a $1000^{\circ} \mathrm{C}$ e, $0,016 \mathrm{~mm}$ para a amostra aquecida a $900^{\circ} \mathrm{C}$, como indicado na tab. 2 .

Tabela 2. Resultados dos testes de tração realizados no Aço API 5L X80.

\begin{tabular}{|c|c|c|}
\hline Temperatura $\left({ }^{\circ} \mathbf{C}\right)$ & Tam. de Grão $(\mathbf{m m})$ & $\boldsymbol{\sigma E s c}(\mathrm{MPa})$ \\
\hline 1100 & $\mathbf{0 , 0 4 8} \pm \mathbf{0 , 0 0 2}$ & $\mathbf{4 4 3 , 1} \pm \mathbf{1 1 , 7 3}$ \\
\hline 1000 & $\mathbf{0 , 0 2 8} \pm \mathbf{0 , 0 0 1}$ & $\mathbf{4 7 3 , 0} \pm \mathbf{5 , 5 2}$ \\
\hline 900 & $\mathbf{0 , 0 1 6} \pm \mathbf{0 , 0 0 1}$ & $\mathbf{5 1 8 , 2} \pm \mathbf{1 0 , 1 6}$ \\
\hline $\mathrm{CR}^{*}$ & $\mathbf{0 , 0 2 0} \pm \mathbf{0 , 0 0 2}$ & $\mathbf{5 4 3 , 9} \pm \mathbf{1 9 , 7 2}$ \\
\hline
\end{tabular}

* Material como recebido 
Diante da observação os corpos de provas representando a ZTA de uma soldagem produzidos em simulador termomecânico, confirma a teoria de que com o distanciamento da zona de fusão os grãos tendem a um refinamento ao mesmo tempo em que, como anteriormente mencionado, vai tendo os valores de $\sigma_{\text {Máx }}$ e $\sigma_{\text {Esc }}$ aumentando, acompanhando o que nos relata a equação de Hall-Petch. Para os valores encontrados na tab. 2, o corpo de prova aquecido a $900^{\circ} \mathrm{C}$, relacionando com os valores do corpo de prova do material CR, há uma diminuição da resistência, justificado pelo fato de que a esta temperatura ocorre parcial recristalização de subestruturas diminuindo o tamanho do grão em relação ao material como recebido, fig. 7(c).

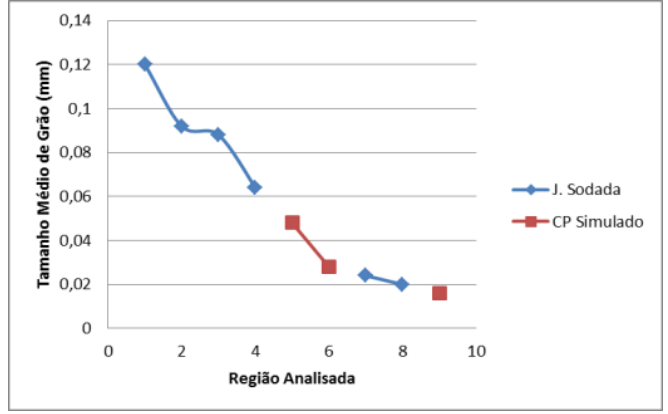

(a)

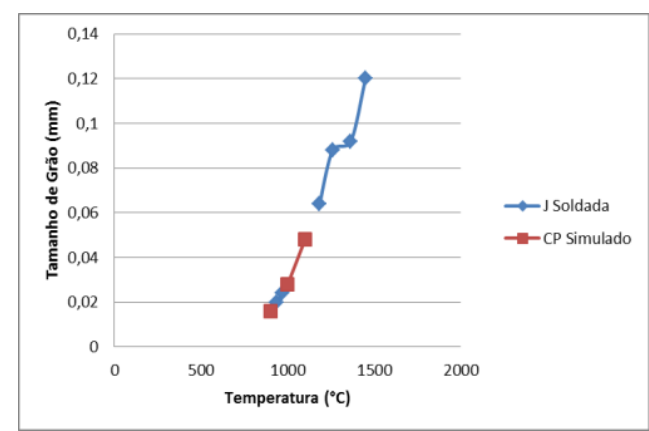

(b)

Figura 8 - Comparação do Tamanho médio dos grãos: (a) da junta soldada; (b) material obtido em simulador.

As microestruturas do material simulado como podem ser vistos nas fig. 8, encaixa-se perfeitamente com a microestrutura da soldagem realizada experimentalmente. A dificuldade maior foi a de observar os grãos refinados da ZTA, próximo ao metal de base, onde acontece um pico de temperatura mais baixo, necessitando de uma melhor atenção por apresentar uma vasta quantidade de sub-grãos motivado pela recristalização do material nesta região, Seabra (1990), e assim dificultando a visibilidade na definição do grão.

\section{CONCLUSÕES}

A metodologia aplicada neste estudo mostrou que é possível a simulação de condições termodinâmicas de pontos discretos da ZTA em corpos de prova que cujas propriedades possam ser obtidas por ensaios tradicionais.

Os resultados observados demonstram a viabilidade do uso do simulador termomecânico para produzir corpos de provas que represente a microestrutura de sub-regiões da ZTA de uma junta soldada e, através de dados obtidos experimentalmente e aplicados na equação empírica de Hall-Petch, podendo prever suas propriedades mecânicas.

Controlando-se as condições termodinâmicas no simulador é possível obter-se materiais com microestrutura e tamanho de grão comparáveis a pontos discretos da zona termicamente afetada (ZTA) que poderá servir de base para modelagem das propriedades mecânicas da junta pelo método de elementos dos volumes finitos.

\section{AGRADECIMENTO}

Ao Programa de Formação de Recursos Humanos em Tecnologia de Equipamentos para atuação no setor de petróleo, gás e biocombustíveis da PETROBRÁS; A SIMISA; Ao Departamento do Curso de Mecânica do IFPE; Ao Instituto Federal de Alagoas; Ao Departamento de Engenharia Mecânica da UFPE, que possibilitam o desenvolvimento deste trabalho.

\section{REFERÊNCIAS}

Gorni, A.A., Estudo traça relações entre microestrutura e propriedades mecânicas em aços estruturais, Corte \& Conformação de Metais - Março 2008.

Silva Jr, L. A.; Yadava, Y. P.; Ferreira, R. A. S. Simulação das transformações de fase em pontos da ZTA de juntas soldadas do aço API $5 L$ X70. In: VI CONGRESSO NACIONAL DE ENGENHARIA MECÂNICA, Campina Grande, Paraiba, Brasil. 2010.

Castro, R. C.; Ferreira, R. A. S.; Pedrosa, I. R. V.; Yadava, Y. P. Effects of termomechanical treatment on the occurrence of Coincident Site Lattice boundaries in High Strength Low Alloy Steel, Open Journal of Metal, Scientific Research Publishing. 2012.

Ginzburg, V.B., Basic Principles of Modeling in Metallurgical Design of Fat-Rolled Steel Products. In: AISE Annual Convention. Proceedings. Pittsburgh, 2003.

Toyoda, M., Fracture Toughness Evaluation of Steel Welds, part I 1988.

Seabra A. L. Estudo Microestrutural da Zona Afetada Pelo Calor (ZAC) de um Aço ARBL Temperado e Revenido. Dissertação (Mestrado em Ciencias dos Materiais - Materiais) - PUC-RJ, Rio de Janeiro, Brasil, 1990. 
NBR 13284, 1995 . Preparação de corpos-de-prova para análise metalográfica - Procedimento. NBR 6152, 2002. Ensaio de tração à temperatura ambiente - Materiais metálicos.

NBR 8108, 1983. Ataque com reativos metalográficos em ligas ferrosas - Procedimento.

\title{
RESPONSABILIDADE AUTORAL
}

"Os autores são os únicos responsáveis pelo conteúdo deste trabalho".

\section{USE OF EQUATION OF THE HALL-PETCH FOR VALIDATION OF SAMPLES PRODUCED IN THERMOMECHANICAL SIMULATOR, TO PREDICT MECHANICAL PROPERTIES OF POINTS OF THE HEAT AFFECTED ZONE IN A WELDED JOINT}

\author{
Roberto de Araújo Alécio, ralecio@gmail.com; \\ Jorge Luís Lauriano Gama, Jorge.lauriano@gmail.com²; \\ Tiago de Sousa Antonino, tiago_antonino@yahoo.com³ \\ Pablo Batista Guimarães, pabloguimaraes@ifpe.recife.edu.br³ \\ José Maria Andrade Barbosa, jmab13@ufpe.br ${ }^{1}$; \\ Yogendra Prasad Yadava, yadava@ufpe.br ${ }^{1}$; \\ Ricardo Artur Sanguinetti Ferreira, ras@ufpe.br¹.

\begin{abstract}
${ }^{1}$ Department of Mechanical Engineering/ Center for Technology and Geosciences/ Federal University of Pernambuco Av. Prof. Moraes Rego, 1235 - Cidade Universitária, CEP: 50670-901, Recife/PE - Brazil.

${ }^{2}$ Course coordinator of Mechanical/Academic Management of Technology and Processes/Federal Institute of Alagoas Av. Barão de Atalaia, s/n - Centro, CEP: 57050-520, Maceió/AL - Brazil.

3Coordination of Mechanical/Academic Department of Control and Industrial Process/ Federal Institute of Pernambuco Av. Prof Luiz Freire, 500, - Cidade Universitária, CEP: 50740-540 Recife/PE - Brazil.
\end{abstract}

\begin{abstract}
:
The heat affected zone (HAZ) of a steel subjected to welding (short region which lies in viziança of the fusion zone where there is a large heating without melting) is sub-divided according to the thermal cycle (peak temperature) that is received. The grain size is a basic microstructural parameters to obtain the mechanical properties, associating the phase transformation diagram with thermal cycle, is observed in the HAZ microstructure with coarse grains after the border region, then a region of fine grain, then a intercritical region and finally a subcritical region that receives less heat than $973 \mathrm{~K}$. The Hall-Petch empirical equation relates the yield stress of a metal with an grain average diameter based on experimental measurements, knowing the constants related to the material of interest. It is the common loss of properties in the HAZ due to undesirable microstructure transformation (embrittlement, precipitation, etc.). based the thermal cycles. A theoretical-experimental methodology that enables the production of microstructures, is being developed in this work, from a cooling induced in thermomechanical simulator, reproducing the thermodynamic conditions of subregions of HAZ ensuring samples with microstructural homogeneity of standard dimensions. The Experimental data are analyzed according to the Hall-Petch empirical equation, so that the variables of yield stress and the friction coefficient of the material are obtained by linear regression with the curve determined from the resistance values and the grain size of body proof produced in the thermomechanical simulator. Based on the microstructural characterization, a welded joint was produced and identified points of the HAZ that are equivalent to bodies of evidence produced in the thermomechanical simulator. Considering the Hall-Petch relationship were compared the simulated stress by the mathematical model with the stresses of the points of the HAZ of the welded joint. The method was satisfactory conclusive therefore showed that the micrographic observation and stress values obtained confirm that defines the literature on the steel studied.
\end{abstract}

Keywords: Hall-Petch, Mechanical properties, thermomechanical simulator, HAZ. 